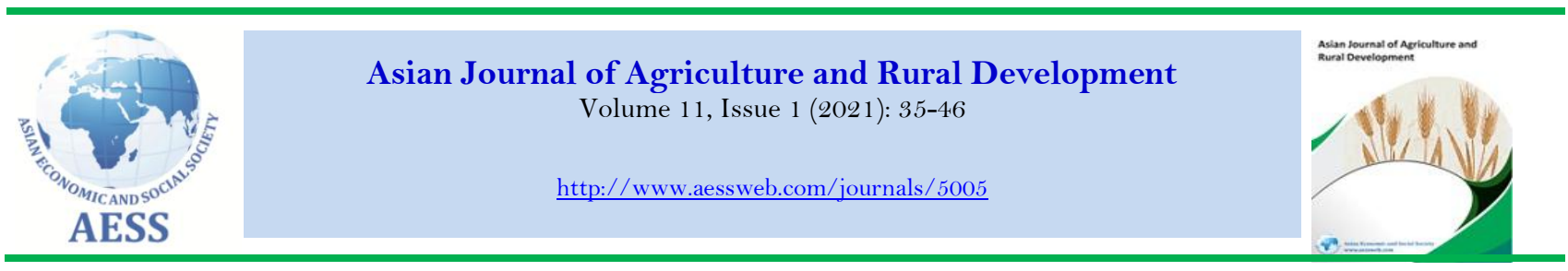

\title{
BIOACTIVE COMPOUNDS AND BIOCHEMICAL AND ANTIOXIDANT PROPERTIES OF SELECTED MINOR INDIGENOUS FRUITS IN BANGLADESH
}

\author{
iD Molla, M.M. ${ }^{a}$ \\ Sabuz, A.A. ${ }^{\text {b }}$ \\ Chowdhury, M.G.F.c \\ Khan, M.H.H ${ }^{d}$ \\ Alam, M. ${ }^{\text {e }}$ \\ Nasrin, T.A.A. ${ }^{f}$ \\ Khatun, A. ${ }^{\mathrm{g}}$ \\ Islam, M.M. ${ }^{\mathrm{h}}$
}

\section{Article History}

Received: 10 November 2020

Revised: 18 December 2020

Accepted: 4 January 2021

Published: 25 January 2021

\section{Keywords}

Minor fruit

Nutritional composition

Minerals

Phytochemicals compound

Antioxidants properties.

\author{
a.b,c,dePostharvest Technology Division, Bangladesh Agricultural Research Institute (BARI), \\ Bangladesh.

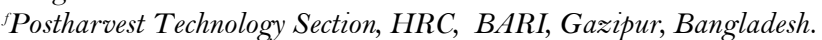 \\ ${ }^{8}$ Institute of Food Science and Technology, BCSIR, Dhaka, Bangladesh. \\ ${ }^{n}$ Nutrition Unit, Bangladesh Agricultural Research Council (BARC), Dhaka, Bangladesh.
}

ه

\begin{abstract}
Minor fruits are a potential source of antinutrients, but there is no complete primary data source in the Bangladeshi context. Therefore, the present study was undertaken to acquire documentation for a database of the composition of selected minor fruits. The total phenolic $(\mathrm{TPH})$, vitamin $\mathrm{C}$, total carotene, and $B$-carotene contents and antioxidant activity of selected minor fruits were determined by 1,1diphenyl-2picryl hydrazyl (DPPH) scavenging and reducing power assays (RPA). Phenolic compounds were assessed using highperformance liquid chromatography coupled with a photodiode array detector and autosampler. Results revealed that minor fruits contain different phytochemicals, particularly TPH, ascorbic acid, total flavonoid (TF), $\beta$-carotene, total carotenoid (TC), and total anthocyanin content (TAC); values ranged, respectively, 0.23-176.50 $\mathrm{mg} \mathrm{GAE} / \mathrm{g}, 16.67-664.92 \mathrm{mg} / 100 \mathrm{~g}, 2.26-150.02 \mathrm{mg} \mathrm{QE} / 100 \mathrm{~g}, 1.41-$ $6897.57 \mu \mathrm{g} / 100 \mathrm{~g}, 1.26-98.24 \mathrm{mg} / 100 \mathrm{~g}$ and $1.15-47.46 \mathrm{mg} / 100 \mathrm{~g}$. In the parameters antioxidant activity, total antioxidant capacity, DPPH, reducing power capacity (RPC), ferric reducing antioxidant power (FRAP), metal chelating capacity (MCC), nitric oxide (NO), and free radical scavenging activity, $\mathrm{IC}_{50}$ ranged 0.01-278.24 $\mu \mathrm{g}$ of ascorbic $\mathrm{acid} / \mathrm{mg}$ of extract, 39.70-250.00\%, 3.21-634.00\%, 0.02-1817.88 $\mu \mathrm{M}$ Fe2SO4/100g, 22.29-210.43\%, 0.02-70.50\%, and 4.98-856.70 $\mu \mathrm{g} / \mathrm{g}$, respectively. Among the identified and quantified phenolic acids, leading examples were gallic acid $(279.06 \mathrm{mg} / 100 \mathrm{~g})$, vanilic acid $(43.77 \mathrm{mg} / 100 \mathrm{~g})$, P-courmaric acid $(178.96 \mathrm{mg} / 100 \mathrm{~g})$, ferulic acid (20.44 $\mathrm{mg} / 100 \mathrm{~g}$ ), and lutein $(91.13 \mu \mathrm{g} / 100 \mathrm{~g}$ ) in aonla, day fruit, elephant apple, and bilimbi. Moreover, all selected minor fruits are rich sources of bioactive, biochemical, and antioxidant compounds with potential for use in therapeutic applications.
\end{abstract}

Contribution/Originality: The study contributes the first logical analysis of selected minor indigenous fruits grown in Bangladesh in terms of phytochemical and antioxidant activities. The nutritional, phytochemical, and antioxidant profiles of these fruits may encourage their consumption rather than that of major and exotic fruits. Pharmaceutical researchers may apply this phytochemical and antioxidant profile for pharmaceutical research purposes. 
DOI: $10.18488 /$ journal.ajard.202 1.111.35.46

$\operatorname{ISSN}(\mathrm{P}):$ 2304-1455/ ISSN(E): 2224-4433

How to cite: Molla, M.M. -- Sabuz, A.A. --- Chowdhury, M.G.F. --- Khan, M.H.H --- Alam, M. --- Nasrin, T.A.A. --- Khatun, A. --- Islam, M.M. (2021). Bioactive Compounds and Biochemical and Antioxidant Properties of Selected Minor Indigenous Fruits in Bangladesh. Asian Journal of Agriculture and Rural Development, 11(1), 35-46. 10.18488/journal.ajard.2021.111.35.46

(C) 2021 Asian Economic and Social Society. All rights reserved.

\section{INTRODUCTION}

Bangladesh is blessed with a great diversity of fruits and, in 2017, was placed sixth in world rankings for tropical fresh fruit production (FAOSTAT, 2019). A significant quantity of tropical fruits are underexploited - generally recognized as indigenous or minor fruits. These fruits are not commercially cultivated and are not frequently found in national or international markets. Usually these fruits are found in home yards, unused high lands, hill tracts, and roadsides. No extra care and farming management are needed to grow these fruits, and that is why people describe these as underutilized minor indigenous fruits in Bangladesh; year on year, these fruit trees are being destroyed. However, these minor fruits can be a good source of micronutrients and phytochemicals. Micronutrients are essential elements in the development of physical growth and help prevent various acute and chronic diseases. Low dietary intake of micronutrient-rich foods, as well as low absorption and lower bioavailability, are the leading causes of micronutrient deficienciy. Phytochemicals with antioxidant capacity naturally present in fruits are of great interest due to their beneficial effects on human health, while regular consumption is associated with reduced risk of developing chronic diseases because they offer protection against oxidative deterioration (Paul et al., 2007). Nowadays, antioxidants are also considered as important as vitamins in the promotion of health and prevention of various diseases linked to reactive oxygen species (ROS), which have been linked to over 100 disorders (Halliwell \& Gutteridge, 2000). Excess generation of ROS causes oxidative stress that damages cellular DNA, lipids, and proteins, leading to the pathogenesis of various diseases including cerebrovascular diseases. Among various antioxidants and phytochemicals, flavonoids, anthocyanin, phenolic acids, and other compounds are linked with natural protective agents, astringents, antibiotics, positive health effects, and antimicrobial properties (Osorio-Esquivel, Álvarez, Dorantes-Álvarez, \& Giusti, 2011). In recent years, interest in antioxidant-rich fruits and their products has been growing in both domestic and international markets because of increasing appreciation of their role in the protection of human health. This has occurred because of their nutritional and antioxidant properties, and also because of the prospects offered to the agricultural and pharmaceutical industries and a promising future source of income for local inhabitants.

A nutrition database is of great importance in addressing the nutritional health benefits of both rural and urban communities. It is essential for the formulation of a national policy on food to have a database on nutritional composition. However, little information in nutrition databases is available for minor fruits. Several studies have been performed on a few fruits only, but these are indiscriminate and have not been scientifically documented or aimed at the consumer. Therefore, documentation, conservation, and revalorization of indigenous knowledge on minor fruits is urgently needed to promote nutritional health for both rural and urban inhabitants. With that in mind, the present research waas undertaken to analyze, document, and popularize the role of phytochemical content in highlighting the phenols, flavonoids, and carotenoids present in selected minor fruits in Bangladesh.

\section{MATERIALS AND METHODS}

\subsection{Materials}

Nine minor indigenous fruits were selected based on peoples' choice and production catchments in Bangladesh, and are shown in Table 1. Analytical grade chemicals and reagents used in this study were procured from SigmaAldrich (Steinheim, Germany).

Table 1. Selected minor indigenous fruits grown in Bangladesh.

\begin{tabular}{c|c|c|c|c}
\hline Sl. no. & Bengali name & English name & Scientific name & Family \\
\hline 1 & Aamlaki & Aonla & Phyllunththus embelica L. & Phyllanthaceae \\
\hline 2 & Amra & Hog plum & Spondias mombin L. & Anacardiaceae \\
\hline 3 & Bilimbi & Cucumber tree & Averrohoa bilimbi L. & Oxalidaceae \\
\hline 4 & Chulta & Elephant apple & Dillenia india L. & Dilleniaceae \\
\hline 5 & Day fall & Day fruit & - & - \\
\hline 6 & Jara lebu & Citron & Citrus medica & Rutaceae \\
\hline 7 & Satkara & Melanesian papeda & Citrus macroptera & Rutaceae \\
\hline 8 & Sour kul & Ber & Ziziphus mauritania & Rhammaceace \\
\hline 9 & Toikar & Toikar & Garcinia pedunculata & Clusiaceae \\
\hline $\begin{array}{l}\text { Source: Project Completion Report, Competitive Research Grant (ID: 328), Bangladesh Agricultural Research Council (BARC), } \\
\text { Farmgate, Dhaka-1215, Bangladesh. }\end{array}$
\end{tabular}




\subsection{Methods}

\subsubsection{Sample Collection and Fruit Extraction}

Fruits were collected from the Regional Agricultural Research Station (RARS), Bangladesh Agricultural Research Institute (BARI), Akbarpur, Moulvibazar, Bangladesh. After collection, the fruits were washed with potable water and surface dried. The fruits were then freeze-dried and milled to powder using a laboratory grinder. Fruit powder of known quantity was extracted in a thermostatic water bath (Vision Scientific Co. Ltd.) at $60^{\circ} \mathrm{C}$ for $1 \mathrm{~h}$ using methanol $(80 \%, \mathrm{v} / \mathrm{v})$ maintaining a fruit:solvent ratio of $1: 20(\mathrm{w} / \mathrm{v})$. The fruit extract was filtered under vacuum, centrifuged at $4000 \times \mathrm{g}$ for $10 \mathrm{~min}$ and the supernatant was collected and kept at $-18^{\circ} \mathrm{C}$ until used for analysis.

\subsubsection{Determination of Physicochemical Parameters}

Physicochemical properties - moisture, protein, ash, total soluble solids (TSS), pH, and titrable acidity - were determined following the method of AOAC (2005). Ascorbic acid, starch, and total sugar content were determined based on the procedure of Ranganna (1995). Edible and non-edible portions of the fruits were measured using the gravimetric method.

\subsubsection{Analysis of Minerals}

Minerals analyzed included sodium, potassium, calcium, magnesium, phosphorus, sulphur, boron, copper, manganese, iron, and zinc. Before quantification, fruits were first wet ashed and then digested in nitric and perchloric acid solution at $320^{\circ} \mathrm{C}$, cooled, diluted to an appropriate concentration, and filtered. This filtrate was then used as the stock solution for further analysis. Atomic absorption spectrophotometry (Model-AA-70ooS, Shimadzu, Tokyo, Japan) was used to assess levels of sodium, iron, copper, zinc, boron, manganese, calcium, and magnesium. Potassium content was measured using flame photometry, while phosphorous and sulphur were assessed by spectrophotometry. Individual minerals were quantified by comparison to the corresponding standard mineral procured from Sigma Chemical Co., USA.

\subsubsection{Determination of Phytochemicals}

\subsubsection{Total Phenolic Content}

Twenty milligrams $(0.02 \mathrm{~g})$ of powder was dissolved in $1 \mathrm{ml}$ of methanol to prepare a stock solution for experiments. A volume of $0.5 \mathrm{ml}$ of the each extract $(100 \mu \mathrm{g} / \mathrm{ml})$ was mixed with $2 \mathrm{ml}$ of Folin-Ciocalteu reagent (diluted 1:10 with deionized water) and neutralized with $4 \mathrm{ml}$ of sodium carbonate solution (7.5\%, w/v). The reaction mixture was incubated at room temperature for $30 \mathrm{~min}$ with intermittent shaking for color development. Absorbance of the colored solution was measured at $765 \mathrm{~nm}$ using double-beam UV-VIS spectrophotometry. Total phenolic content was determined from the linear equation of a standard curve prepared with gallic acid. Determination of total phenolic content in the extracts was determined according to the Folin-Ciocalteu method (Ough \& Amerine, 1988), with gallic acid (GAE) as the standard and expressed (mg) as gallic acid equivalents (GAE)/g of extract (Aoshima, Hirata, \& Ayabe, 2007).

\subsubsection{Determination of Total Flavonoid Content}

Total flavonoid content (TFC) of the fruit powder was determined by the aluminium chloride method (Chang, Yang, Wen, \& Chern, 2002) with slight modifications: $0.5 \mathrm{ml}$ of sample solution was mixed with $1.5 \mathrm{ml}$ of methanol. To this mixture $0.1 \mathrm{ml}$ of $10 \%$ aluminium chloride and $0.1 \mathrm{ml}$ of $1 \mathrm{M}$ potassium acetate were added. The final volume was made up to $5 \mathrm{ml}$ by adding $2.8 \mathrm{ml}$ of distilled water, and the reaction was left for 30 min at room temperature. Absorbance of the solution was measured at $415 \mathrm{~nm}$ and expressed as $\mathrm{mg} \mathrm{QE} / \mathrm{g}$ extract. TFC was calculated from the calibration curve of quercetin plotted (Figure 1). The curve obtained was found to be linear with equation $y=0.0035$ $+0.0056 x$, and the correlation coefficient was found to be $R^{2}=0.9993$. TFC was expressed as mg quercetin equivalents per gram of extract (mg QE/g extract).

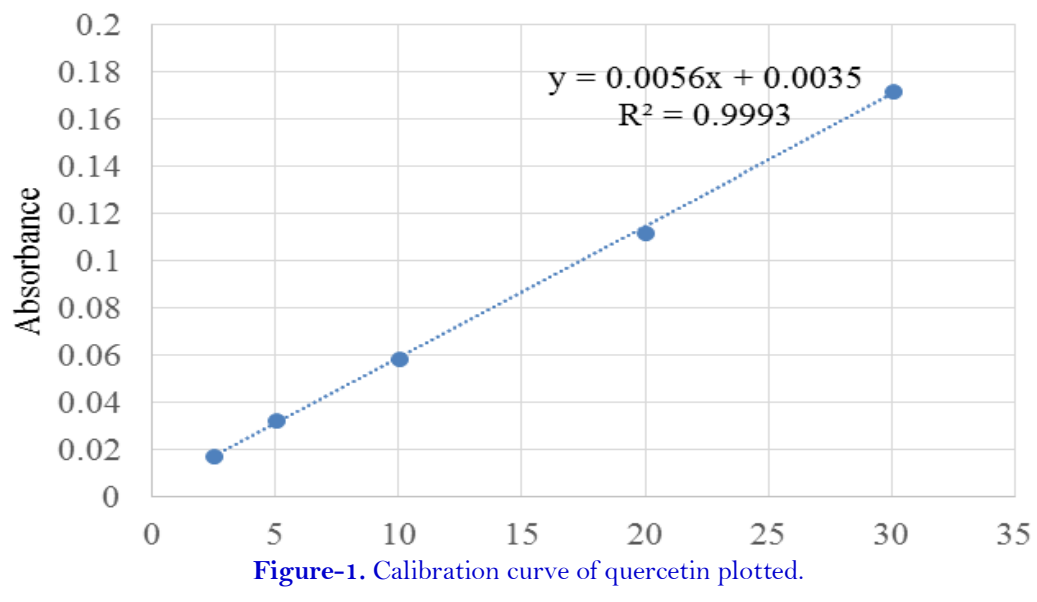




\subsubsection{Determination of Total Anthocyanin (TA)}

The method was adapted from Burgos et al. (2013), the concentration of TA being calculated using the molar extinction coefficient and molecular weight of malvidin-3-p-coumaroyl-glucoside for blue-violet pigments $(545 \mathrm{~nm}$, $\left.3.02 \times 10^{4} \mathrm{l} / \mathrm{mol} / \mathrm{cm}, 718.5 \mathrm{~g} / \mathrm{mol}\right)$, pelargonidin-3-glucoside for red pigments $\left(515 \mathrm{~nm}, 2.73 \times 10^{4} 1 / \mathrm{mol} / \mathrm{cm}, 486.5\right.$ $\mathrm{g} / \mathrm{mol}$ ), and cyanidin-3-glucoside for purple pigments $\left(535 \mathrm{~nm}, 3.43 \times 10^{4} \mathrm{l} / \mathrm{mol} / \mathrm{cm}, 449.2 \mathrm{~g} / \mathrm{mol}\right.$ ). Results were expressed as $\mathrm{mg} / 100 \mathrm{~g} \mathrm{DW}$.

\subsubsection{Determination of Total Carotenoid Content}

Total carotenoid was determined according to the method described by Thaipong, Boonprakob, Crosby, CisnerosZevallos, and Byrne (2006). Absorbance was measured at $\lambda 470 \mathrm{~nm}$. Each extract was dissolved in n-hexane proanalysis. B-carotene solution in various concentrations was used as standard carotenoid compound and the standard curve. A linear regression equation of the standard curve was used for calculating total carotenoid content, and expressed as beta-carotene equivalents per $100 \mathrm{~g}$ of extract $(\mathrm{mg} / 100 \mathrm{~g})$.

\subsubsection{Determination of $\beta$-Carotene Content}

$\beta$-Carotene content was determined using the procedure of Holden et al. (1999), with values expressed as $\mu \mathrm{g} / 100 \mathrm{~g}$ of fruit extract.

\subsubsection{Determination of Antioxidant Activity}

2.2.5.1. Total Atioxidant Activity

Total antioxidant activity was assessed using the phosphomolybdenum system according to the technique described by Prieto, Pineda, and Aguilar (1999), and results expressed as micrograms ascorbic acid (AA) per gram $(\mu \mathrm{g} / \mathrm{ml})$ of the sample.

\subsubsection{Reducing Power Assay}

The reducing power of the fruit extract was assessed using the approach of Guo, Saravanakumar, and Wang (2018). Ascorbic acid was used as the standard for the preparation of the calibration curve.

\subsubsection{FRAP}

FRAP activity was measured following the scheme outlined by Benzie and Strain (1996). A standard curve was made using ferrous sulphate aqueous solution (1-10 mM) and FRAP values were expressed as $\mu \mathrm{M} \mathrm{Fe}$ (II)/100g of the sample.

\subsubsection{DPPH Radical Scavenging Activity (DPPH-RSA) and IC $C_{50}$}

DPPH-RSA was evaluated by measuring the inhibition rate following the procedure described by Brand-Williams, Cuvelier, and Berset (1995), with modification. Exactly $0.1 \mathrm{ml}$ of extract was placed in a Falcon tube and $1.4 \mathrm{ml}$ of methanolic solution of DPPH added. The mix was left to rest for $30 \mathrm{~min}$ in the dark and absorbance at $517 \mathrm{~nm}$ was measured against a blank. The results are expressed as percentage radical scavenging activity:

$$
\text { DPPH radical scavenging activity }(\%)=\frac{A_{O}-A_{s}}{A_{o}} \times 100
$$

where $A_{o}$ is absorbance of blank and $A_{s}$ is absorbance of sample extract. The inhibition curves were then prepared and $\mathrm{IC}_{50}$ values calculated (Brand-Williams et al., 1995). BHT was the positive control.

\subsubsection{5. $M C C$}

MCC was determined based on Bahadori, Zengin, Bahadori, Dinparast, and Movahhedin (2018), with minor modification. Briefly, $2 \mathrm{ml}$ of extract was placed in a glass tube to which $0.05 \mathrm{ml}$ of ferrous chloride $(2 \mathrm{mM}), 3.7 \mathrm{ml}$ of distilled water, and $0.2 \mathrm{ml}$ of ferrozine $(5 \mathrm{mM})$ were added. After $20 \mathrm{~min}$ of incubation under atmospheric conditions, absorbance was read at $562 \mathrm{~nm}$ against a blank. The following formula was applied to calculate MCC:

$$
\text { Metal chelating capacity }(\%)=\frac{\text { Absorbance }(\text { control })-\text { Absorbance }(\text { sample })}{\text { Absorbance }(\text { control })} \times 100
$$

\subsubsection{Nitric Oxide (NO) Radical Scavenging Activity}

Nitric oxide radical quenching activity was determined according to the procedure of Bogucka-Kocka, Zidorn, Kasprzycka, Szymczak, and Szewczyk (2018), with some modification. In a glass tube, $0.5 \mathrm{ml}$ of extract was mixed with $2 \mathrm{ml}$ of sodium nitroprusside $(10 \mathrm{mM})$. This was followed by incubation under atmospheric conditions for $1 \mathrm{~h}$. Thereafter, $0.5 \mathrm{ml}$ of the incubated mixture was transferred to another centrifuge tube, Griess reagent (0.5 ml) was added, and the mixture rested for $30 \mathrm{~min}$. Absorbance of the solution was read at $540 \mathrm{~nm}$ against a blank. The following formula was employed to calculate the result, expressed as percentage inhibition:

$$
\text { Inhibition }(\%)=\frac{\text { Absorbance }(\text { control })-\text { Absorbance }(\text { sample })}{\text { Absorbance }(\text { control })} \times 100
$$

\subsubsection{Assessment of Phenolic Acids by HPLC}

Phenolic compounds were assessed according to the method of Pandey and Negi (2018), with some adjustment, using high-performance liquid chromatography (HPLC; Shimadzu SPD-M10A) coupled with a photodiode array 
detector and autosampler at 280 and $320 \mathrm{~nm}$. Separation was achieved using a C18 column $\left(250 \times 4.6 \mathrm{~mm}^{2}\right)$ of $5 \mu \mathrm{m}$ particle size at room temperature. The mobile phase was $1 \%$ acetic acid (A) and $80 \%$ acetonitrile in $\mathrm{A}$ (B). The following gradient was used: $0.01-35 \mathrm{~min}, \mathrm{O} \%$ of $\mathrm{B} ; 35-40 \mathrm{~min}, 50 \%$ of $\mathrm{B} ; 40-45 \mathrm{~min}, 100 \%$ of $\mathrm{B}$; and $45-60 \mathrm{~min}, \mathrm{O} \%$ of $\mathrm{B}$. The flow rate was $1 \mathrm{ml} / \mathrm{min}$ and the injection capacity was $20 \mu \mathrm{l}$. Six phenolic standards (P-courmaric acid, gallic acid, vanilic acid, caffeic acid, ferulic acid, and lutein) were used for identification of respective phenolics, and quantification was accomplished using a standard curve.

\section{STATISTICAL ANALYSIS}

Data obtained for each analysis were expressed in duplicate as means \pm standard deviation. Data were analyzed by one-way ANOVA with post hoc using Tukey's multiple comparisons test. Significance was defined at the 95\% confidence level. Statistical analysis and data processing were performed using the software SPSS 17.0 (IBM Inc., New York).

\section{RESULTS AND DISCUSSION}

\subsection{Biochemical Properties}

4.1.1. Crude Protein

Analysis of crude protein content of selected minor fruits is shown in Table 2. It will be seen that in all fruits this is statistically significant, with levels of crude protein ranging from 2.39 to $4.10 \%$ (Table 2). The highest levels of protein were found in citron $(4.10 \%)$ and satkara $(4.06 \%)$. Total protein obtained from the Bangladeshi commercial mango fruits amrapali, chausa, fazlee, gopalbhog, himsagor, and langra was found to be 0.14, 0.26, 0.21, 0.07, 1.11, and $1.18 \mathrm{~g} / 100 \mathrm{~g}$, respectively (Ara, Motalab, Uddin, Fakhruddin, \& Saha, 2014). The crude protein content of Indian minor fruits Aegle marmalose Correa, Cordia myxa L., Zizipus mauritiana Lam, Averrhoa bilimbi L., and Grewia asiatica Mast weas recorded as 2.2, 1.9, 1.04, 1.3, and $1.09 \mathrm{~g} / 100 \mathrm{~g}$, respectively (Mitra, Pathak, \& Chakraborty, 2008). The crude protein content found in our minor indigenous fruits was higher than that found by both Mitra et al. (2008) and Ara et al. (2014).

\subsubsection{Ash}

The ash content of the nine selected minor fruits was statistically significant, ranging from 1.40 to $3.78 \%$, the maximum (3.78\%) being recorded in citron (Table 2$)$. The highest ash content found in the present study is comparable to the findings of Shukla, Dubey, Jain, and Kumar (2001), who reported that Adansonia digitata contain $3.31 \%$ ash.

\subsubsection{Total Sugar}

Total sugar content of the nine selected minor fruit ranged from 2.83 to $5.64 \%$. All values were statistically highly significant, with the maximum content recorded for hog plum (5.64\%) and the minimum for aonla (2.83\%) (Table 2 ). Values for blilimbi, elephant apple, day fruit, citron, satkara, ber and toikar were 4.07, 4.13, 4.20, 4.27, 3.91, 4.35, and $4.02 \%$, respectively. The results obtained from our study are supported by the findings of Ara et al. (2014), who reported that the total sugar content of commercial mango varieties ranged from 4.27 to $4.83 \%$.

\subsection{Starch}

Starch is a major carbohydrate source of immense economic and nutritional value. It is essential that the food industry search for new starch sources to meet the requirements of both the food processing industry and consumers. Table 2 shows selected minor fruits containing higher starch levels and all statistically highly significant differences. The highest starch content $(8.70 \%)$ was recorded for day fruit and the lowest for satkara (3.44\%). The high total sugar content in day fruit may be due to enzymatic hydrolysis of starch to sugar (Nelson, 1944). The lower yield of starch in satkara is probably due to its increased respiration directly by genetical induced.

\subsection{Moisture}

The moisture content of the selected minor fruits varied. The data show that the moisture content of the selected minor fruits was statistically significant $(P<0.05)$, ranging from 76.62 to $80.73 \%$ - maximum in citron $(80.73 \%)$ and minimum in aonla $(76.62 \%)$ (Table 2 ). The results obtained from the study are comparable to those of Parveen and Khatkar (2015able 3), who reported aonla (variety Desi) with $81.26 \%$ moisture content.

\subsection{Acidity}

Acidity varied highly significantly among the selected minor fruits, ranging from 0.79 to $3.58 \%$ - the maximum was $3.58 \%$ in day fruit and the minimum in citron (Table 2). The results obtained from this study confirm that the acidity of the selected minor fruits is much higher than that found by Akhter et al. (2010) for langra (0.68\%) and chausa $(0.63 \%)$ commercial mango cultivars in Bangladesh. The reason for this discrepancy may be that most minor fruits are citrus fruits with variation in maturity stage, harvesting time, soil management, and cultural practices. 
Table 2. Biochemical and mineral composition of selected minor fruits.

\begin{tabular}{|c|c|c|c|c|c|c|c|c|c|}
\hline \multirow[t]{2}{*}{ Parameter } & \multicolumn{9}{|c|}{ Minor fruits } \\
\hline & Aonla & Hog plum & Bilimbi & Elephant apple & Day fruit & Citron & Satkara & Ber (sour) & Toikar \\
\hline Crude protein $(\%)$ & $\begin{array}{l}3.71 \pm \\
0.03 \mathrm{ab}\end{array}$ & $\begin{array}{c}3.80 \pm \\
0.10 \mathrm{ab} \\
\end{array}$ & $\begin{array}{c}3.70 \pm \\
0.20 \mathrm{ab} \\
\end{array}$ & $\begin{array}{c}3.26 \pm \\
0.15 \mathrm{bc} \\
\end{array}$ & $\begin{array}{c}3.04 \pm \\
0.01\end{array}$ & $\begin{array}{l}4.10 \pm \\
0.10 \mathrm{a} \\
\end{array}$ & $\begin{array}{l}4.06 \pm \\
0.02 \mathrm{a} \\
\end{array}$ & $\begin{array}{c}3.18 \pm \\
0.09 \mathrm{bc}\end{array}$ & $\begin{array}{l}2.39 \pm \\
0.57 \mathrm{~d} \\
\end{array}$ \\
\hline $\operatorname{Ash}(\%)$ & $\begin{array}{l}1.92 \pm \\
0.01 \mathrm{f}\end{array}$ & $\begin{array}{l}2.23 \pm \\
0.02 \mathrm{e}\end{array}$ & $\begin{array}{c}3.33 \pm \\
0.01 \mathrm{bcd} \\
\end{array}$ & $\begin{array}{l}3.45 \pm \\
0.02 \mathrm{bc}\end{array}$ & $\begin{array}{l}1.40 \pm \\
0.10 \mathrm{~g}\end{array}$ & $\begin{array}{l}3.78 \pm \\
0.02 \mathrm{a}\end{array}$ & $\begin{array}{l}3.31 \pm \\
0.10 \mathrm{~cd}\end{array}$ & $\begin{array}{l}3.21 \pm \\
0.10 \mathrm{~d}\end{array}$ & $\begin{array}{l}3.49 \pm \\
0.02 \mathrm{~b}\end{array}$ \\
\hline Total sugars (\%) & $\begin{array}{l}2.83 \pm \\
0.01 \mathrm{~d}\end{array}$ & $\begin{array}{l}5.64 \pm \\
0.03 \mathrm{a}\end{array}$ & $\begin{array}{l}4.07 \pm \\
0.06 \mathrm{bc}\end{array}$ & $\begin{array}{l}4.13 \pm \\
0.04 \mathrm{bc}\end{array}$ & $\begin{array}{l}4.20 \pm \\
0.10 b c\end{array}$ & $\begin{array}{l}4.27 \pm \\
0.03 \mathrm{bc}\end{array}$ & $\begin{array}{l}3.91 \pm \\
0.06 \mathrm{c}\end{array}$ & $\begin{array}{l}4.35 \pm \\
0.40 b\end{array}$ & $\begin{array}{l}4.02 \pm \\
0.02 \mathrm{bc}\end{array}$ \\
\hline Starch $(\%)$ & $\begin{array}{l}5.51 \pm \\
0.27 \mathrm{~b}\end{array}$ & $\begin{array}{l}4.45 \pm \\
0.08 \mathrm{~cd}\end{array}$ & $\begin{array}{l}4.13 \pm \\
0.02 \mathrm{de}\end{array}$ & $\begin{array}{l}4.71 \pm \\
0.03 \mathrm{~cd}\end{array}$ & $\begin{array}{l}8.70 \pm \\
0.20 \mathrm{a} \\
\end{array}$ & $\begin{array}{l}3.69 \pm \\
0.58 \mathrm{ef}\end{array}$ & $\begin{array}{l}3.44 \pm \\
0.03 f \\
\end{array}$ & $\begin{array}{l}4.15 \pm \\
0.02 \mathrm{de}\end{array}$ & $\begin{array}{l}4.85 \pm \\
0.07 \mathrm{c}\end{array}$ \\
\hline Moisture (\%) & $\begin{array}{c}76.62 \pm \\
0.10 \mathrm{~g} \\
\end{array}$ & $\begin{array}{c}78.44 \pm \\
0.11 \mathrm{ef} \\
\end{array}$ & $\begin{array}{c}77.77 \pm \\
0.10 f \\
\end{array}$ & $\begin{array}{l}79.02 \pm \\
\text { 0.13def } \\
\end{array}$ & $\begin{array}{l}79.49 \pm \\
\text { O.54bcd } \\
\end{array}$ & $\begin{array}{c}80.73 \pm \\
0.25 \mathrm{a} \\
\end{array}$ & $\begin{array}{l}80.33 \pm \\
0.09 \mathrm{ab} \\
\end{array}$ & $\begin{array}{l}78.61 \pm \\
0.48 \text { def } \\
\end{array}$ & $\begin{array}{l}79.82 \pm \\
0.57 \mathrm{abc} \\
\end{array}$ \\
\hline Acidity (\%) & $\begin{array}{c}1.82 \pm \\
0.02 \mathrm{de}\end{array}$ & $\begin{array}{l}2.29 \pm \\
0.02 \mathrm{~b}\end{array}$ & $\begin{array}{c}1.84 \pm \\
0.02 \text { cde }\end{array}$ & $\begin{array}{c}1.92 \pm \\
0.03 \mathrm{~cd}\end{array}$ & $\begin{array}{l}3.58 \pm \\
0.03 \mathrm{a}\end{array}$ & $\begin{array}{c}0.79 \pm \\
0.02 f\end{array}$ & $\begin{array}{l}1.78 \pm \\
0.10 \mathrm{e}\end{array}$ & $\begin{array}{l}1.95 \pm \\
0.05 \mathrm{c}\end{array}$ & $\begin{array}{l}2.40 \pm \\
0.02 \mathrm{~b}\end{array}$ \\
\hline $\mathrm{pH}$ & $\begin{array}{l}2.65 \pm \\
0.02 \mathrm{e}\end{array}$ & $\begin{array}{l}2.94 \pm \\
0.00 \mathrm{~cd}\end{array}$ & $\begin{array}{r}2.14 \pm \\
0.02 f \\
\end{array}$ & $\begin{array}{l}3.29 \pm \\
0.02 \mathrm{~b}\end{array}$ & $\begin{array}{l}2.63 \pm \\
0.10 \mathrm{e} \\
\end{array}$ & $\begin{array}{l}3.53 \pm \\
0.03 \mathrm{a} \\
\end{array}$ & $\begin{array}{l}3.58 \pm \\
0.09 \mathrm{a} \\
\end{array}$ & $\begin{array}{l}2.88 \pm \\
0.04 \mathrm{~d}\end{array}$ & $\begin{array}{l}3.09 \pm \\
0.02 \mathrm{c}\end{array}$ \\
\hline $\mathrm{TSS}\left({ }^{\circ} \mathrm{B}\right)$ & $\begin{array}{c}11.40 \pm \\
0.02 \mathrm{a} \\
\end{array}$ & $\begin{array}{c}10.91 \pm \\
0.01 \mathrm{~b}\end{array}$ & $\begin{array}{c}10.18 \pm \\
0.02 \mathrm{c}\end{array}$ & $\begin{array}{l}9.17 \pm \\
0.00 d\end{array}$ & $\begin{array}{l}7.01 \pm \\
0.01 \mathrm{~g}\end{array}$ & $\begin{array}{l}7.54 \pm \\
0.25 \mathrm{f}\end{array}$ & $\begin{array}{l}8.50 \pm \\
0.10 \mathrm{e}\end{array}$ & $\begin{array}{c}10.44 \pm \\
0.05 \mathrm{c}\end{array}$ & $\begin{array}{l}9.20 \pm \\
0.02 \mathrm{~d}\end{array}$ \\
\hline Edible (\%) & $\begin{array}{c}84.62 \pm \\
0.02 \mathrm{~d}\end{array}$ & $\begin{array}{c}47.59 \pm \\
0.3 f\end{array}$ & $\begin{array}{c}98.00 \pm \\
1.00 \mathrm{a} \\
\end{array}$ & $\begin{array}{l}79.03 \pm \\
0.02 \mathrm{e}\end{array}$ & $\begin{array}{c}90.95 \pm \\
0.03 \mathrm{~b}\end{array}$ & $\begin{array}{c}79.77 \pm \\
1.59 \mathrm{e} \\
\end{array}$ & $\begin{array}{c}44.70 \pm \\
0.20 \mathrm{~g}\end{array}$ & $\begin{array}{c}88.37 \pm \\
0.31 \mathrm{c} \\
\end{array}$ & $\begin{array}{c}84.01 \pm \\
0.01 \mathrm{~d} \\
\end{array}$ \\
\hline Non-edible (\%) & $\begin{array}{c}15.38 \pm \\
0.02 \\
\end{array}$ & $\begin{array}{c}50.50 \pm \\
0.16 \mathrm{a} \\
\end{array}$ & $\begin{array}{c}2.00 \pm \\
1.00 f \\
\end{array}$ & $\begin{array}{c}18.02 \pm \\
1.01 \mathrm{c} \\
\end{array}$ & $\begin{array}{l}8.01 \pm \\
0.01 \mathrm{e}\end{array}$ & $\begin{array}{c}20.05 \pm \\
1.20 \mathrm{~b} \\
\end{array}$ & $\begin{array}{c}50.01 \pm \\
0.09 \mathrm{a} \\
\end{array}$ & $\begin{array}{l}9.30 \pm \\
0.09 \mathrm{e}\end{array}$ & $\begin{array}{c}15.33 \pm \\
0.03 \mathrm{~d} \\
\end{array}$ \\
\hline \multicolumn{10}{|l|}{ Minerals } \\
\hline $\mathrm{Ca}$ & $\begin{array}{l}0.25 \pm \\
0.00 \mathrm{e}\end{array}$ & $\begin{array}{l}0.27 \pm \\
0.00 \mathrm{e}\end{array}$ & $\begin{array}{l}0.58 \pm \\
0.00 \mathrm{~cd}\end{array}$ & $\begin{array}{l}0.54 \pm \\
0.00 d\end{array}$ & $\begin{array}{l}0.67 \pm \\
0.02 \mathrm{c}\end{array}$ & $\begin{array}{l}0.82 \pm \\
0.05 \mathrm{~b}\end{array}$ & $\begin{array}{l}0.55 \pm \\
0.00 d\end{array}$ & $\begin{array}{l}1.02 \pm \\
0.05 \mathrm{a}\end{array}$ & $\begin{array}{l}0.87 \pm \\
0.06 \mathrm{~b}\end{array}$ \\
\hline $\mathrm{Mg}$ & $\begin{array}{l}0.15 \pm \\
0.01 \mathrm{e} \\
\end{array}$ & $\begin{array}{l}0.14 \pm \\
0.00 \mathrm{e}\end{array}$ & $\begin{array}{l}0.31 \pm \\
0.00 d\end{array}$ & $\begin{array}{l}0.28 \pm \\
0.00 d\end{array}$ & $\begin{array}{l}0.29 \pm \\
0.02 \mathrm{~d}\end{array}$ & $\begin{array}{l}0.37 \pm \\
0.02 \mathrm{c} \\
\end{array}$ & $\begin{array}{l}0.29 \pm \\
0.00 d\end{array}$ & $\begin{array}{l}0.57 \pm \\
0.03 \mathrm{a} \\
\end{array}$ & $\begin{array}{l}0.49 \pm \\
0.03 \mathrm{~b}\end{array}$ \\
\hline K & $\begin{array}{l}0.93 \pm \\
0.00 c d\end{array}$ & $\begin{array}{l}0.93 \pm \\
\text { 0.oocd }\end{array}$ & $\begin{array}{c}1.07 \pm \\
0.01 \mathrm{ab}\end{array}$ & $\begin{array}{l}1.08 \pm \\
0.00 a\end{array}$ & $\begin{array}{l}0.40 \pm \\
0.02 \mathrm{f}\end{array}$ & $\begin{array}{l}0.64 \pm \\
0.04 \mathrm{e}\end{array}$ & $\begin{array}{l}0.99 \pm \\
0.00 b c\end{array}$ & $\begin{array}{l}0.87 \pm \\
0.03 \mathrm{~d}\end{array}$ & $\begin{array}{l}0.37 \pm \\
0.04 f\end{array}$ \\
\hline $\mathrm{Na}$ & $\begin{array}{l}0.32 \pm \\
0.01 \mathrm{a} \\
\end{array}$ & $\begin{array}{c}0.16 \pm \\
\text { 0.oode } \\
\end{array}$ & $\begin{array}{l}0.18 \pm \\
0.01 \mathrm{~cd}\end{array}$ & $\begin{array}{l}0.19 \pm \\
0.00 c\end{array}$ & $\begin{array}{c}0.12 \pm \\
0.00 f\end{array}$ & $\begin{array}{l}0.08 \pm \\
0.01 \mathrm{~g}\end{array}$ & $\begin{array}{l}0.15 \pm \\
0.00 \mathrm{e}\end{array}$ & $\begin{array}{l}0.22 \pm \\
0.00 \mathrm{~b}\end{array}$ & $\begin{array}{l}0.18 \pm \\
0.00 \mathrm{~cd} \\
\end{array}$ \\
\hline $\mathrm{P}$ & $\begin{array}{l}0.42 \pm \\
0.00 a\end{array}$ & $\begin{array}{c}0.42 \pm \\
0.00 a\end{array}$ & $\begin{array}{l}0.11 \pm \\
0.00 e f\end{array}$ & $\begin{array}{l}0.31 \pm \\
0.01 \mathrm{c}\end{array}$ & $\begin{array}{l}0.07 \pm \\
0.01 \mathrm{~g}\end{array}$ & $\begin{array}{c}0.10 \pm \\
0.00 f\end{array}$ & $\begin{array}{l}0.34 \pm \\
0.00 b\end{array}$ & $\begin{array}{l}0.12 \pm \\
0.00 \mathrm{e}\end{array}$ & $\begin{array}{l}0.21 \pm \\
0.00 \mathrm{~d}\end{array}$ \\
\hline $\mathrm{S}$ & $\begin{array}{l}0.07 \pm \\
0.00 b\end{array}$ & $\begin{array}{l}0.07 \pm \\
0.00 b\end{array}$ & $\begin{array}{l}0.06 \pm \\
0.00 b\end{array}$ & $\begin{array}{l}0.05 \pm \\
0.01 \mathrm{~b}\end{array}$ & $\begin{array}{l}0.02 \pm \\
0.00 \mathrm{c}\end{array}$ & $\begin{array}{l}0.02 \pm \\
0.00 \mathrm{c}\end{array}$ & $\begin{array}{l}0.06 \pm \\
0.00 b\end{array}$ & $\begin{array}{l}0.36 \pm \\
0.00 \mathrm{a} \\
\end{array}$ & $\begin{array}{l}0.36 \pm \\
0.00 \mathrm{a} \\
\end{array}$ \\
\hline B & $\begin{array}{c}68.04 \pm \\
2.16 \mathrm{a} \\
\end{array}$ & $\begin{array}{l}4.82 \pm \\
0.02 \mathrm{de}\end{array}$ & $\begin{array}{c}14.31 \pm \\
0.02 \mathrm{C}\end{array}$ & $\begin{array}{c}26.30 \pm \\
0.14 \mathrm{~b}\end{array}$ & $\begin{array}{c}13.40 \pm \\
3.51 \mathrm{c}\end{array}$ & $\begin{array}{l}8.03 \pm \\
0.30 d\end{array}$ & $\begin{array}{l}1.36 \pm \\
1.01 \mathrm{e} \\
\end{array}$ & $\begin{array}{l}8.60 \pm \\
0.26 \mathrm{~d} \\
\end{array}$ & $\begin{array}{l}6.38 \pm \\
0.40 d \\
\end{array}$ \\
\hline $\mathrm{Cu}$ & $\begin{array}{l}4.82 \pm \\
0.02 \mathrm{~h}\end{array}$ & $\begin{array}{c}128.30 \pm \\
0.10 \mathrm{c}\end{array}$ & $\begin{array}{c}107.53 \pm \\
0.97 \mathrm{e}\end{array}$ & $\begin{array}{c}157.77 \pm \\
0.24 \mathrm{~b}\end{array}$ & $\begin{array}{c}11.67 \pm \\
0.30 \mathrm{~g}\end{array}$ & $\begin{array}{c}114.22 \pm \\
5.02 \mathrm{~d}\end{array}$ & $\begin{array}{c}62.61 \pm \\
0.02 f\end{array}$ & $\begin{array}{c}155.78 \pm \\
1.31 \mathrm{~b}\end{array}$ & $\begin{array}{c}395.52 \pm \\
1.77 \mathrm{a}\end{array}$ \\
\hline
\end{tabular}




\begin{tabular}{l|c|c|c|c|c|c|c|c|c}
\hline $\mathrm{Fe}$ & $90.12 \pm$ & $68.32 \pm$ & $57.48 \pm$ & $83.91 \pm$ & $180.99 \pm$ & $73.47 \pm$ & $35.30 \pm$ & $155.78 \pm$ & $238.26 \pm$ \\
& $0.02 \mathrm{~d}$ & $0.06 \mathrm{e}$ & $0.21 \mathrm{f}$ & $0.05 \mathrm{~d}$ & $5.86 \mathrm{~b}$ & $2.98 \mathrm{e}$ & $0.03 \mathrm{~g}$ & $1.31 \mathrm{c}$ & $1.07 \mathrm{a}$ \\
\hline $\mathrm{Mn}$ & $47.92 \pm$ & $27.52 \pm$ & $81.78 \pm$ & $151.19 \pm$ & $116.68 \pm$ & $16.06 \pm$ & $11.22 \pm$ & $93.84 \pm$ & $19.99 \pm$ \\
& $0.01 \mathrm{e}$ & $0.01 \mathrm{f}$ & $0.03 \mathrm{~d}$ & $0.99 \mathrm{a}$ & $6.38 \mathrm{~b}$ & $0.60 \mathrm{gh}$ & $0.03 \mathrm{~h}$ & $0.78 \mathrm{c}$ & $0.99 \mathrm{~g}$ \\
\hline $\mathrm{Zn}$ & $18.84 \pm$ & $13.16 \pm$ & $20.00 \pm$ & $17.84 \pm$ & $23.34 \pm$ & $11.20 \pm$ & $10.14 \pm$ & $10.73 \pm$ & $22.33 \pm$ \\
& $0.02 \mathrm{ab}$ & $0.15 \mathrm{~cd}$ & $2.00 \mathrm{ab}$ & $0.28 \mathrm{bc}$ & $0.60 \mathrm{a}$ & $4.42 \mathrm{~d}$ & $0.01 \mathrm{~d}$ & $0.50 \mathrm{~d}$ & $0.57 \mathrm{ab}$ \\
\hline
\end{tabular}

Note: Values are mean \pm standard deviation $(n=3) ; \mathrm{Ca}, \mathrm{Mg}, \mathrm{K}, \mathrm{Na}, \mathrm{P}$, and $\mathrm{S}$ expressed as $\mathrm{mg} \% ; \mathrm{B}, \mathrm{Cu}, \mathrm{Fe}, \mathrm{Mn}$, and $\mathrm{Zn}$ expressed as ppm. All values are means of triplicate determinations $\pm \mathrm{SD}$. Means within column with different letters a, b, c, d, e, f, g, indicate significant result ( $\mathrm{p}<0.05)$.

Table 3. Phytochemical and antioxidant properties of selected minor indigenous fruits

\begin{tabular}{|c|c|c|c|c|c|c|c|c|c|}
\hline \multirow[t]{2}{*}{ Parameter } & \multicolumn{9}{|c|}{ Minor fruits } \\
\hline & Aonla & Hog plum & Bilimbi & Elephant apple & Day fruit & Citron & Satkara & Ber (sour) & Toikar \\
\hline \multicolumn{10}{|l|}{ Phytochemical propertrties } \\
\hline TPH (mg GAE/g) & $\begin{array}{c}2.10 \pm \\
0.09 \\
\end{array}$ & $\begin{array}{l}0.23 \pm \\
0.02 \mathrm{e}\end{array}$ & $\begin{array}{c}105.92 \pm \\
0.14 \mathrm{~b} \\
\end{array}$ & $\begin{array}{c}62.85 \pm \\
0.05 \mathrm{c} \\
\end{array}$ & $\begin{array}{c}176.50 \pm \\
2.42 \mathrm{a} \\
\end{array}$ & $\begin{array}{c}50.00 \pm \\
0.08 \mathrm{~d}\end{array}$ & $\begin{array}{l}1.58 \pm \\
0.08 \mathrm{e}\end{array}$ & $\begin{array}{l}2.04 \pm \\
0.06 \mathrm{e}\end{array}$ & $\begin{array}{l}1.81 \pm \\
0.07 \mathrm{e}\end{array}$ \\
\hline $\mathrm{TF}(\mathrm{mg} \mathrm{QE} / \mathrm{g})$ & $\begin{array}{c}45.04 \pm \\
2.47 \mathrm{~b} \\
\end{array}$ & $\begin{array}{l}2.84 \pm \\
0.11 \mathrm{~d} \\
\end{array}$ & $\begin{array}{c}150.02 \pm \\
1.17 \mathrm{a} \\
\end{array}$ & $\begin{array}{c}26.16 \pm \\
1.96 \mathrm{c} \\
\end{array}$ & $\begin{array}{c}27.00 \pm \\
3.04 \mathrm{c} \\
\end{array}$ & $\begin{array}{c}27.85 \pm \\
1.05 \mathrm{c} \\
\end{array}$ & $\begin{array}{c}23.38 \pm \\
1.25 \mathrm{c} \\
\end{array}$ & $\begin{array}{l}2.26 \pm \\
0.09 \mathrm{~d} \\
\end{array}$ & $\begin{array}{c}18.33 \pm \\
1.12 \\
\end{array}$ \\
\hline Total $\beta$-carotene $(\mu \mathrm{g} / 100 \mathrm{~g})$ & $\begin{array}{c}6897.57 \\
\pm 0.09 \mathrm{a} \\
\end{array}$ & $\begin{array}{l}1737.11 \\
\pm 3.27 \mathrm{~d} \\
\end{array}$ & $\begin{array}{c}39.33 \pm \\
1.52 \mathrm{f} \\
\end{array}$ & $\begin{array}{c}1.95 \pm \\
0.05 f\end{array}$ & $\begin{array}{l}1.41 \pm \\
0.04 \mathrm{~g}\end{array}$ & $\begin{array}{l}9.58 \pm \\
0.09 \mathrm{~g}\end{array}$ & $\begin{array}{c}2318.44 \pm \\
5.60 \mathrm{~b} \\
\end{array}$ & $\begin{array}{c}549.12 \pm \\
6.42 \mathrm{e} \\
\end{array}$ & $\begin{array}{c}1815.88 \pm \\
3.62 \mathrm{C} \\
\end{array}$ \\
\hline $\mathrm{TC}(\mathrm{mg} / 100 \mathrm{~g})$ & $\begin{array}{c}83.73 \pm \\
0.39 \mathrm{c}\end{array}$ & $\begin{array}{l}68.45 \pm \\
0.12 \mathrm{e} \\
\end{array}$ & $\begin{array}{l}4.70 \pm \\
0.30 f\end{array}$ & $\begin{array}{l}1.58 \pm \\
0.09 \mathrm{~g}\end{array}$ & $\begin{array}{l}1.26 \pm \\
0.23 \mathrm{~g}\end{array}$ & $\begin{array}{l}2.07 \pm \\
0.11 \mathrm{~g}\end{array}$ & $\begin{array}{c}93.91 \pm \\
0.80 \mathrm{~b}\end{array}$ & $\begin{array}{c}74.89 \pm \\
1.12 \mathrm{~d}\end{array}$ & $\begin{array}{c}98.24 \pm \\
0.09 \mathrm{a}\end{array}$ \\
\hline TA (mg/100 g) & $\begin{array}{c}15.53 \pm \\
0.03 \mathrm{~b} \\
\end{array}$ & $\begin{array}{l}5.11 \pm \\
0.01 \mathrm{~d}\end{array}$ & $\begin{array}{c}47.46 \pm \\
0.22 \mathrm{a} \\
\end{array}$ & $\begin{array}{l}1.74 \pm \\
0.23 \mathrm{e} \\
\end{array}$ & $\begin{array}{l}1.15 \pm \\
0.14 \mathrm{f}\end{array}$ & $\begin{array}{l}1.19 \pm \\
0.03 \mathrm{f}\end{array}$ & $\begin{array}{l}6.25 \pm \\
0.04 \mathrm{c} \\
\end{array}$ & $\begin{array}{l}1.66 \pm \\
0.01 \mathrm{e}\end{array}$ & $\begin{array}{l}6.24 \pm \\
0.01 \mathrm{c} \\
\end{array}$ \\
\hline $\mathrm{AA}(\mathrm{mg} / 100 \mathrm{~g})$ & $\begin{array}{c}664.92 \pm \\
1.00 \mathrm{a} \\
\end{array}$ & $\begin{array}{c}67.90 \pm \\
0.05 \mathrm{e}\end{array}$ & $\begin{array}{c}28.41 \pm \\
0.09\end{array}$ & $\begin{array}{c}40.29 \pm \\
0.11 \mathrm{f}\end{array}$ & $\begin{array}{c}16.67 \pm \\
0.53 \mathrm{i} \\
\end{array}$ & $\begin{array}{c}24.28 \pm \\
0.61 \mathrm{~h} \\
\end{array}$ & $\begin{array}{c}210.43 \pm \\
0.02 \mathrm{~b}\end{array}$ & $\begin{array}{c}160.12 \pm \\
0.11 \mathrm{c}\end{array}$ & $\begin{array}{c}142.81 \pm \\
0.99 \mathrm{~d}\end{array}$ \\
\hline \multicolumn{10}{|l|}{ Antioxidant activity } \\
\hline $\mathrm{TAC}(\mu \mathrm{g} \mathrm{AA} / \mathrm{g})$ & $\begin{array}{c}0.02 \pm \\
0.00 f \\
\end{array}$ & $\begin{array}{c}0.01 \pm \\
0.00 f \\
\end{array}$ & $\begin{array}{c}108.06 \pm \\
0.45 \mathrm{c} \\
\end{array}$ & $\begin{array}{c}85.90 \pm \\
0.03 \mathrm{~d}\end{array}$ & $\begin{array}{c}122.32 \pm \\
0.01 \mathrm{~b} \\
\end{array}$ & $\begin{array}{c}278.24 \pm \\
0.03 \mathrm{a} \\
\end{array}$ & $\begin{array}{c}0.01 \pm \\
0.00 f\end{array}$ & $\begin{array}{c}0.02 \pm \\
0.00 f\end{array}$ & $\begin{array}{l}1.14 \pm \\
0.02 \mathrm{e}\end{array}$ \\
\hline$\overline{\mathrm{DPPH}}(\%)$ & $\begin{array}{c}250.00 \pm \\
10.00 \mathrm{~b}\end{array}$ & $\begin{array}{c}314.00 \pm \\
5.00 \mathrm{a}\end{array}$ & $\begin{array}{c}73.27 \pm \\
0.25 \mathrm{~d}\end{array}$ & $\begin{array}{c}68.40 \pm \\
4.60 d\end{array}$ & $\begin{array}{c}87.70 \pm \\
0.49 \mathrm{c}\end{array}$ & $\begin{array}{c}70.20 \pm \\
0.19 \mathrm{~d}\end{array}$ & $\begin{array}{c}39.70 \pm \\
0.20 \mathrm{e}\end{array}$ & $\begin{array}{c}41.66 \pm \\
0.76 \mathrm{e}\end{array}$ & $\begin{array}{c}45.00 \pm \\
5.00 \mathrm{e}\end{array}$ \\
\hline $\mathrm{RPA}(\mu \mathrm{g} \mathrm{AA} / \mathrm{g})$ & $\begin{array}{c}490.50 \pm \\
0.50 b\end{array}$ & $\begin{array}{c}634.00 \pm \\
1.00 \mathrm{a}\end{array}$ & $\begin{array}{c}18.71 \pm \\
0.01 \mathrm{e}\end{array}$ & $\begin{array}{l}5.89 \pm \\
0.02 f\end{array}$ & $\begin{array}{c}3.21 \pm \\
0.01 \mathrm{f}\end{array}$ & $\begin{array}{c}3.85 \pm \\
0.02 \mathrm{f}\end{array}$ & $\begin{array}{c}93.00 \pm \\
2.00 d\end{array}$ & $\begin{array}{c}105.66 \pm \\
12.09 \mathrm{~d}\end{array}$ & $\begin{array}{c}130.00 \pm \\
5.00 \mathrm{c}\end{array}$ \\
\hline$\overline{\mathrm{FRAP}}\left(\mu \mathrm{M} \mathrm{Fe}_{2} \mathrm{SO}_{4} / 100 \mathrm{~g}\right)$ & $\begin{array}{c}38.01 \pm \\
9.99 \mathrm{e}\end{array}$ & $\begin{array}{c}4.87 \pm \\
0.1 \text { of }\end{array}$ & $\begin{array}{c}1817.88 \pm \\
3.62 \mathrm{~b}\end{array}$ & $\begin{array}{c}2325 \pm \\
6.09 \mathrm{a}\end{array}$ & $\begin{array}{c}1213.46 \pm \\
0.47 \mathrm{~d}\end{array}$ & $\begin{array}{c}1247.50 \pm \\
3.25 \mathrm{c}\end{array}$ & $\begin{array}{c}0.35 \pm \\
0.05 \mathrm{f}\end{array}$ & $\begin{array}{c}1.86 \pm \\
0.20 f\end{array}$ & $\begin{array}{c}0.02 \pm \\
0.00 f\end{array}$ \\
\hline $\mathrm{MCC}(\%)$ & $\begin{array}{c}33.89 \pm \\
0.02 \mathrm{~h}\end{array}$ & $\begin{array}{c}182.39 \pm \\
0.06 \mathrm{c}\end{array}$ & $\begin{array}{c}192.97 \pm \\
1.01 \mathrm{~b}\end{array}$ & $\begin{array}{c}22.29 \pm \\
0.09 \mathrm{i}\end{array}$ & $\begin{array}{c}97.56 \pm \\
0.10 d\end{array}$ & $\begin{array}{c}210.43 \pm \\
0.02 \mathrm{a}\end{array}$ & $\begin{array}{c}40.76 \pm \\
0.09 \mathrm{~g}\end{array}$ & $\begin{array}{c}72.00 \pm \\
1.00 \mathrm{e}\end{array}$ & $\begin{array}{c}59.51 \pm \\
0.01 \mathrm{f}\end{array}$ \\
\hline NO (\%) & $\begin{array}{l}7.97 \pm \\
0.10 \mathrm{e}\end{array}$ & $\begin{array}{l}0.01 \pm \\
0.00 g\end{array}$ & $\begin{array}{c}70.50 \pm \\
1.00 \mathrm{~b}\end{array}$ & $\begin{array}{c}40.18 \pm \\
0.03 d\end{array}$ & $\begin{array}{c}41.65 \pm \\
0.02 \mathrm{c}\end{array}$ & $\begin{array}{c}178.87 \pm \\
0.02 \mathrm{a}\end{array}$ & $\begin{array}{l}1.09 \pm \\
0.06 f\end{array}$ & $\begin{array}{l}0.02 \pm \\
0.009\end{array}$ & $\begin{array}{c}1.27 \pm \\
0.06 f\end{array}$ \\
\hline $\mathrm{IC}_{50}(\mu \mathrm{g} / \mathrm{g})$ & $\begin{array}{c}255.20 \pm \\
0.20 d\end{array}$ & $\begin{array}{c}856.70 \pm \\
0.03 \mathrm{a}\end{array}$ & $\begin{array}{c}30.36 \pm \\
1.32 \mathrm{~g}\end{array}$ & $\begin{array}{c}4.98 \pm \\
0.24 \mathrm{i}\end{array}$ & $\begin{array}{c}49.57 \pm \\
0.50 f\end{array}$ & $\begin{array}{c}11.26 \pm \\
0.26 \mathrm{~h}\end{array}$ & $\begin{array}{c}362.00 \pm \\
0.50 \mathrm{c}\end{array}$ & $\begin{array}{c}242.78 \pm \\
0.03 \mathrm{e}\end{array}$ & $\begin{array}{c}504.00 \pm \\
1.00 \mathrm{~b}\end{array}$ \\
\hline
\end{tabular}

Note: All values are means of triplicate determinations \pm SD. Means within columns with different letters a, b, c, d, e, f, g,h, i indicate significant result ( $(\mathrm{p}<0.05)$. 
4.5. $p H$

$\mathrm{pH}$ levels of the selected minor fruits are shown in Table 2; these ranged from 2.14 to 3.58. pH levels were highly significant, with maxima of 3.53 and 3.58 for citron and satkara, respectively, and a minimum of 2.14 for bilimbi (Averrhoa bilimbi L.). pH levels showed an inverse relationship to acidity, with a minimum of $0.79 \%$ for citron but a maximum of 3.53. A similar observation was also made by Ara et al. (2014) for major mango fruit commercial cultivars. Variation in $\mathrm{pH}$ among selected minor fruits may be due to variation in their internal metabolism and ripening behavior.

\subsection{TSS}

TSS include carbohydrates, organic acids, proteins, fats, and minerals. TSS denotes a fresh weight of 10-20\%, and increases with fruit maturity in regard to the production of a less acidic and sweeter fruit. In our study, TSS of the selected fruits varied significantly among species with a maximum of $11.40^{\circ} \mathrm{B}$ for aonla and a minimum for day fruit $\left(7.01^{\circ} \mathrm{B}\right)$. The results obtained from our study confirmed that there was an inverse relationship between TSS and acidity. The acidity of $1.82 \%$ in aonla was influenced by TSS $\left(11.40^{\circ} \mathrm{B}\right)$ (Table 2$)$. Likewise, another relationship was found between TSS and fruit maturity, with increase in TSS following fruit maturity (no maturity data are shown here). For example, in the initial stage, the taste of aonla appears to be astringent but, when fully matured, it becomes sweeter with increasing TSS.

\subsection{Edible Percentage}

Edible percentage differed significantly among the selected fruit cultivars, as shown in Table 2 . The highest edible percentage was calculated at 98.0 in bilimbi (Avverhoa bilimbi L.); all of the fruit, except the pericarp, is used for consumption. The lowest edible percentage was recorded at 44.7 for satakara, due to removal of peel during preprocessing for consumption. However, the edible percentage of the selected minor fruits ranged from 44.7 to 98.0, being much higher than in major fruits (Ara et al., 2014).

\subsection{Non-Edible Percentage}

The non-edible percentage of selected minor fruits ranged from 2.0 to 50.5. The lowest (2.00\%) non-edible percentage was found in bilimbi while the highest recorded were 50.5 and 50.0, in hog plum and satkara, resoectively, due to their natural fruit structure and peel, because most of the peel goes to waste during preprocessing.

\subsection{Mineral Profiling}

Minerals are the inorganic components present in foodstuff as ash when food is burned. Generally macro- and microminerals are present in foodstuffs and play important metabolic roles in body functions (Reilly, 2002), and contribute to our daily diet. In our study, 11 minerals were assessed from the selected minor fruits (Table 2$)$. It will be seen that these contained a significant range of macrominerals including sodium (0.08-0.32 mg\%), potassium (0.37$1.08 \mathrm{mg} \%$ ), calcium (0.25-1.02 mg\%), magnesium (0.14-0.57 mg\%), and phosphorus (0.07-0.42 mg\%); and considerable amounts of microminerals including sulphur (0.02-0.36 mg\%), boron (1.36-68.04 ppm), copper (4.82-395.52 ppm), iron (35.30-238.26 ppm), manganese (11.22-151.19 ppm), and zinc (10.14-23.34 ppm). Our study indicates that sour ber is a rich source of $\mathrm{Ca}, \mathrm{Mg}$, and $\mathrm{S}$; aonla is a rich source of $\mathrm{Na}$ and $\mathrm{P}, \operatorname{hog}$ plum of $\mathrm{P}$, and elephant apple of $\mathrm{K}$ and $\mathrm{Mn}$. Toikar is a rich source of $\mathrm{Cu}$ and $\mathrm{Fe}$, and day fruit of $\mathrm{Zn}$. In comparison with Indian minor fruits, our selected minor fruits possessed lower amounts of various minerals. It is claimed in different studies that soil fertility, genetic factors, fertilizers, and geographical conditions play significant roles in the mineral content of fruits (Tiburski, Rosenthal, Deliza, de Oliveira Godoy, \& Pacheco, 2011).

\subsection{Phytochemical properties}

Phytochemical components reputedly have the ability to lower the prevalence of various degenerative diseases such as cancer, heart attack, and cardiovascular disease, by blocking the activity of free radicals. The results recorded for phytochemical analyses of total phenolics, flavonoids, carotenoids, $\beta$-carotene, ascorbic acid, and anthocyanin are shown in Table 3.

\subsubsection{Total Phenolic Content}

Total phenolic content of the selected minor fruits ranged from 0.23 to $176.50 \mathrm{mg} \mathrm{GAE} / \mathrm{g}$ (Table 3). The maximum was recorded for day fruit $(176.50 \mathrm{mg} \mathrm{GAE} / \mathrm{g}$ ) and the minimum in hog plum (0.23 $\mathrm{mg} \mathrm{GAE} / \mathrm{g})$. Guava, pineapple, soursop, passion, acerola camarinha, and mango recorded a polyphenol content of 83.1, 21.7, 84.3, 20.2, 580.1, 492.8, and 260.21 mg GAE/100g, respectively (Kuskoski, García Asuero, Morales Millán, \& Fett, 2006; Tiburski et al., 2011).

\subsubsection{TFC}

Flavonoids are regarded as a low-molecular-weight substances in foodstuffs and boost antioxidant activity. Their content depends on the levels of polyphenols and geographical location. The value of total flavonoids found in the minor fruits studied ranged from 2.26 to $150.02 \mathrm{mg} \mathrm{QE} / \mathrm{g}$ (Table 3). In our study, bilimbi ranked highest for flavonoid content $(150.0259 \mathrm{mg} \mathrm{QE} / \mathrm{g}$ ) while the minimum was recorded as $2.26 \mathrm{mg} \mathrm{QE} / \mathrm{g}$ in ber. This variation is somewhat similar to previous reports for exotic fruits: Osorio-Esquivel et al. (2011) found a TFC of $13.45-68.79 \mathrm{mg} / 100 \mathrm{~g}$ in Opuntia joconostle. Since these fruits possessed a fair amount of flavonoids like other exotic fruits, its consumption would help to contribute to add antioxidants to our daily diet. 


\subsubsection{Total Carotenoid Content}

It is evidenced from different studies that carotenoid plays a crucial role in human nutrition and health, and can lessen the risks of cancer and heart disease because of the activity of provitamin A (Tiburski et al., 2011). In this study, total carotenoid content of the selected fruits ranged from 1.26 to $98.24 \mathrm{mg} / 100 \mathrm{~g}$ (Table 3). The total carotenoids contained in the selected fruit are comparable to reports published elsewhere: Shajib et al. (2013) reported carotenoid levels of $149 \mu \mathrm{g} / 100 \mathrm{~g}$ for wood apple, $161 \mu \mathrm{g} / 100 \mathrm{~g}$ for orboroi, and $218 \mu \mathrm{g} / 100 \mathrm{~g}$ for Burmese grape.

\subsubsection{Total $\beta$-Carotene Content}

Total $\beta$-carotene content of the selected minor fruits ranged from 1.41 to $6897.57 \mu \mathrm{g} / 100 \mathrm{~g}$. The lowest $\boldsymbol{\beta}$-carotene content was calculated as $1.41 \mu \mathrm{g} / 100 \mathrm{~g}$ in day fruit and the maximum in aonla $(6897.57 \mu \mathrm{g} / 100 \mathrm{~g})$ (Table 3). Comparatively higher values for $\beta$-carotene were reported by Tiburski et al. (2011) for yellow mombin pulp (314 $\mu \mathrm{g} / 100 \mathrm{~g})$. It is evidenced that carotenoid content in fruits depends on various factors including soil conditions, maturity stage, enzymes, phenolic content, and genetics (Leong \& Oey, 2012).

\subsubsection{Ascorbic Acid Content}

Ascorbic acid is an important bioactive compound and is considered the most powerful antioxidant in foodstuffs; regular intake lowers the risk of cancer in the human body (Almeida et al., 2011). In the selected fruits it ranged from 16.67 to $664.90 \mathrm{mg} / 100 \mathrm{~g}$, with a maximum in aonla $(664.90 \mathrm{mg} / 100 \mathrm{~g})$ and a minimum in day fruit $(16.67 \mathrm{mg} / 100 \mathrm{~g})$ on a fresh weight basis (Table 3). These levels are much higher than in the report of Shajib et al. (2013) for monkey jack (14 mg/100 g), Burmese grape (12.1 mg/100 g), orboroi (20.8 mg/100 g), karanda (9.5 mg/100 g), mangosteem $(14.4 \mathrm{mg} / 100 \mathrm{~g})$, blackberry $(25.7 \mathrm{mg} / 100 \mathrm{~g})$, flacourtia $(25.6 \mathrm{mg} / 100 \mathrm{~g})$, rochelle $(3.7 \mathrm{mg} / 100 \mathrm{~g})$, and takituki (27.8 $\mathrm{mg} / 100 \mathrm{~g}$ ). According to Jukes (1974), the recommended daily intake of vitamin C (ascorbic acid) required to prevent scurvy in adults is about $10 \mathrm{mg}$, which indicates that the current study found a level of ascorbic acid in selected fruits exceeding that necessary to prevent scurvy, with a daily intake level of $100 \mathrm{~g}$.

\subsubsection{Anthocyanin Content}

One of the key bioactive compounds in foodstuffs is anthocyanin, which shows potent antioxidant capacity. The anthocyanin content of our selected minor fruits ranged from 1.15 to $47.46 \mathrm{mg} / 100 \mathrm{~g}$ (Table 3 ). The maximum content noted was $47.46 \mathrm{mg} / 100 \mathrm{~g}$ in bilimbi (Averrhoa bilimbi L.) and the minimum in day fruit (1.15 mg/100 g). Moreover, comparatively similar results were found for some tropical fruits (red grapes, $27 \mathrm{mg} / 100 \mathrm{~g}$; cherries, $112 \mathrm{mg} / 100 \mathrm{~g}$; strawberries, $21 \mathrm{mg} / 100 \mathrm{~g}$; and red raspberries, $92 \mathrm{mg} / 100 \mathrm{~g}$ ) (Wu et al., 2006).

\subsection{Evaluation of Antioxidant Activity}

Foodstuffs with high antioxidant properties play a crucial role in the inhibition of ROS-mediated diseases (Dutta \& Ray, 2020).

\subsubsection{Total Antioxidant Capacity}

In this investigation, the antioxidant capacity of the selected minor fruits was assessed by an assortment of different tests. It can be seen that all fruits exhibited potent antioxidant capacity, ranging from 0.01 to $278.24 \mu \mathrm{g} \mathrm{AA} / \mathrm{g}$ (Table $3)$. It is interesting that citron possessed a higher amount of antioxidant capacity $(278.24 \mu \mathrm{g} \mathrm{AA} / \mathrm{g})$ than other fruits.

\subsection{2. DPPH Radical Scavenging Activity}

DPPH is a relatively stable free radical scavenger that donates hydrogen protons to unpaired electrons to convert them into paired ones. It is noted that DPPH radical scavenging activity of the selected minor fruits ranged from 39.7 to $314.0 \%$ (Table 3 ). The maximal DPPH was calculated as $314.0 \%$ in hog plum and the minimum in satkara (39.7\%). However, all selected fruits in our study showed a strong capacity to scavenge free radicals.

\subsection{3. $R P A$}

The RPA of the selected minor fruits ranged from 3.21 to $634.00 \mu \mathrm{g} \mathrm{AA} / \mathrm{mg}$. The maximum value of the reducing power assay was calculated as $634.00 \mu \mathrm{g} \mathrm{AA} / \mathrm{mg}$ in hog plum while the lowest was $3.21 \mu \mathrm{g} \mathrm{AA} / \mathrm{mg}$ in day fruit (Table 3).

\subsection{4. $I C_{50}$}

The $\mathrm{IC}_{50}$ is a widely accepted method used to assess the antioxidant activity of foodstuffs, and its value is expected to be lower for higher free radical quenching ability (Sathyanarayanan, Chandran, Thankarajan, Abrahamse, \& Thangaraj, 2018). In this study, $\mathrm{IC}_{50}$ values ranged from 4.98 to $504.0 \mu \mathrm{g} / \mathrm{g}$ while the maximum value was found in hog plum $(856.7 \mu \mathrm{g} / \mathrm{g})$ and the minimum in elephant apple $(4.98 \mu \mathrm{g} / \mathrm{g})$ (Table 3$)$. Our results indicate that hog plum has potential antioxidant capacity due to its lower value of $\mathrm{IC}_{50}(4.98 \pm 0.24 \mu \mathrm{g} / \mathrm{g})$, which may be due to the presence of significant amounts of phenolics and flavonoids. This finding is also corroborated by the research of Sathyanarayanan et al. (2018).

\subsection{5. $M C C$}

MCC of the selected minor fruits ranged from 22.29 to $210.43 \%$ (Table 3), which indicates the ability of minor fruits to reduce different metallic ions and create a stable chemical bond to counteract free radicals. Recent studies have 
reported that the redox properties of phenolic species enable them to work as reducing agents, by donating hydrogen and quenching singlet oxygen showing antioxidant activity and chelating metal ions (Elfalleh et al., 2011).

\subsection{6. FRAP}

The FRAP values found ranged from 0.35 to $2325.0 \mu \mathrm{M} \mathrm{Fe}_{2} \mathrm{SO}_{4} / 100 \mathrm{~g}$ (Table 3). Our results show a high FRAP value for elephant apple $\left(2325.0 \mu \mathrm{M} \mathrm{Fe}_{2} \mathrm{SO}_{4} / 100 \mathrm{~g}\right)$, demonstrating that phenolic composites are among the leading contributors to the high antioxidant properties of this fruit. Previous reports evidenced that fruits with high phenolics can react with free radicals to form a stable product that blocks the radical chain reaction (Sathyanarayanan et al., 2018).

\subsection{7. NO Radical Scavenging Activity}

NO radical scavenging activity of the selected minor fruits ranged from 0.02 to $178.87 \%$. The results revealed that citron is a rich source of $\mathrm{NO}$ radical scavenging activity, recording the highest value of $178.87 \%$, the lowest value being $0.02 \%$ in sour ber (Table 3$)$. Thus, our study reported high NO radical foraging activity (178.87 $\pm 0.02 \%)$, which may be due to the presence of different polyphenolic substances in citron.

\subsection{Phenolic Compounds}

HPLC was used for analysis of six major phenolic acids and matching to the respective standards. Phenolic acid levels in the selected minor fruits ranged $0.94-279.0 \mathrm{mg} / 100 \mathrm{~g}$ for gallic acid, $0.01-43.77 \mathrm{mg} / 100 \mathrm{~g}$ for vanillic acid, $0.16-178.96 \mathrm{mg} / 100 \mathrm{~g}$ for P-courmaric acid, $0.01-3.04 \mathrm{mg} / 100 \mathrm{~g}$ for caffeic acid, $0.01-20.44 \mathrm{mg} / 100 \mathrm{~g}$ for ferulic acid, and $12.03-97.35 \mu \mathrm{g} / 100 \mathrm{~g}$ for lutein. These results indicate that the leading phenolic compounds were gallic and ferulic acids for aonla; gallic acid for hog plum; gallic, P-courmaric, ferulic, and vanilic acids for bilimbi; gallic, P-courmaric, ferulic, and caffeic acids for elephant apple; gallic, caffeic, and P-courmaric acids for day fruit; P-courmaric and gallic acids for citron; gallic acid for satkara; P-courmaric and gallic acids for ber; and ferulic and caffeic acidc for toikar (Table 4).

Table 4. Phenolic acid content of selected minor indigenous fruits.

\begin{tabular}{l|c|c|c|c|c|c|c|c|c}
\hline Parameter & \multicolumn{10}{c}{ Minor fruits } \\
\cline { 2 - 12 } & Aonla & $\begin{array}{c}\text { Hog } \\
\text { plum }\end{array}$ & Bilimbi & $\begin{array}{c}\text { Elephant } \\
\text { apple }\end{array}$ & $\begin{array}{c}\text { Day } \\
\text { fruit }\end{array}$ & Citron & Satkara & $\begin{array}{c}\text { Ber } \\
(\text { sour })\end{array}$ & Toikar \\
\hline Gallic acid & 279.06 & 58.33 & 82.01 & $38.14 \pm$ & $3.62 \pm$ & $0.94 \pm$ & $66.00 \pm$ & $26.50 \pm$ & $62.74 \pm$ \\
$(\mathrm{mg} / 100 \mathrm{~g})$ & $\pm 2.00 \mathrm{a}$ & $\pm 0.33 \mathrm{~d}$ & $\pm 3.01 \mathrm{~b}$ & $0.15 \mathrm{e}$ & $0.01 \mathrm{~g}$ & $0.02 \mathrm{~g}$ & $0.52 \mathrm{c}$ & $1.17 \mathrm{f}$ & $0.74 \mathrm{c}$ \\
\hline Vanilic acid & $43.77 \pm$ & $42.02 \pm$ & $70.50 \pm$ & 0.01 & $0.04 \pm$ & $0.02 \pm$ & $4.04 \pm$ & $2.46 \pm$ & $0.03 \pm$ \\
$(\mathrm{mg} / 100 \mathrm{~g})$ & $0.15 \mathrm{~b}$ & $0.02 \mathrm{~b}$ & $2.43 \mathrm{a}$ & $\pm 0.00 \mathrm{~d}$ & $0.01 \mathrm{~d}$ & $0.01 \mathrm{~d}$ & $0.04 \mathrm{c}$ & $0.05 \mathrm{c}$ & $0.01 \mathrm{~d}$ \\
\hline $\mathrm{p}-\mathrm{courmaric}$ & $1.06 \pm$ & $0.96 \pm$ & $13.16 \pm$ & $11.51 \pm$ & $178.96 \pm$ & $19.26 \pm$ & $3.00 \pm$ & $38.14 \pm$ & $0.16 \pm$ \\
$(\mathrm{mg} / 100 \mathrm{~g})$ & $0.03 \mathrm{e}$ & $0.02 \mathrm{e}$ & $0.17 \mathrm{~d}$ & $0.07 \mathrm{~d}$ & $4.13 \mathrm{a}$ & $1.54 \mathrm{c}$ & $0.50 \mathrm{e}$ & $0.74 \mathrm{~b}$ & $0.01 \mathrm{e}$ \\
\hline Caffeic acid & $0.09 \pm$ & $0.01 \pm$ & $0.05 \pm$ & $3.04 \pm$ & $0.45 \pm$ & $0.01 \pm$ & $0.10 \pm$ & $0.02 \pm$ & $0.02 \pm$ \\
$(\mathrm{mg} / 100 \mathrm{~g})$ & $0.08 \mathrm{c}$ & $0.00 \mathrm{c}$ & $0.01 \mathrm{c}$ & $0.05 \mathrm{a}$ & $0.01 \mathrm{~b}$ & $0.00 \mathrm{c}$ & $0.05 \mathrm{c}$ & $0.01 \mathrm{c}$ & $0.01 \mathrm{c}$ \\
\hline Ferulic acid & $5.54 \pm$ & $0.21 \pm$ & $9.59 \pm$ & $20.44 \pm$ & $0.01 \pm$ & $0.01 \pm$ & $0.01 \pm$ & $0.20 \pm$ & $0.21 \pm$ \\
$(\mathrm{mg} / 100 \mathrm{~g})$ & $0.03 \mathrm{c}$ & $0.01 \mathrm{~d}$ & $0.06 \mathrm{~b}$ & $0.05 \mathrm{a}$ & $0.00 \mathrm{e}$ & $0.00 \mathrm{e}$ & $0.00 \mathrm{e}$ & $0.15 \mathrm{~d}$ & $0.20 \mathrm{~d}$ \\
\hline Lutein acid & $12.03 \pm$ & $29.74 \pm$ & $91.13 \pm$ & $97.35 \pm$ & $71.33 \pm$ & $71.33 \pm$ & $12.62 \pm$ & $14.85 \pm$ & $16.20 \pm$ \\
$(\mu \mathrm{g} / 100 \mathrm{~g})$ & $0.04 \mathrm{f}$ & $0.11 \mathrm{~d}$ & $3.80 \mathrm{~b}$ & $0.35 \mathrm{a}$ & $0.33 \mathrm{c}$ & $0.33 \mathrm{c}$ & $0.12 \mathrm{ef}$ & $0.03 \mathrm{ef}$ & $0.10 \mathrm{e}$ \\
\hline
\end{tabular}

\section{CONCLUSION}

This study is the first to provide details regarding the biochemical properties, minerals, bioactive compounds, antioxidant activities, and phenolic compounds of nine key minor indigenous fruits grown in Bangladesh. The results indicate that aonla is a rich source of ascorbic acid $(664.92 \mathrm{mg} / 100 \mathrm{~g})$, total $\beta$-carotene $(6897.57 \mu \mathrm{g} / 100 \mathrm{~g})$, and RPA $(634.00 \mu \mathrm{AA} / \mathrm{g})$; bilimbi is a rich source of total flavonoids $(150.02 \mathrm{mg} \mathrm{QE} / \mathrm{g})$, total anthocyanin $(47.46 \mathrm{mg} / 100 \mathrm{~g})$, and $\operatorname{FRAP}\left(1817.88 \mu \mathrm{M} \mathrm{Fe}_{2} \mathrm{SO}_{4} / 100 \mathrm{~g}\right)$; citron is a rich source of TAC (278.24 $\left.\mu \mathrm{AA} / \mathrm{g}\right), \mathrm{MCC}(210.43 \%)$, and $\mathrm{NO}(178.87 \%)$; hog plum is a rich source of DPPH (314.0\%); and day fruit is a rich source of TPH (176.50 mg GAE/g). The findings suggest that all minor fruits studied possess levels of phytochemicals that may be applied as raw material for nutritional and pharmaceutical usage.

Funding: This work was supported by the National Agricultural Technology Program (NATP), Phase- II, Competitive Research Grant sub-project (Grant Number: CRG-328), Bangladesh Agricultural Research Council (BARC), Dhaka, Bangladesh.

Competing Interests: The authors declare that they have no competing interests.

Contributors/Acknowledgement: Authors thankfully acknowledged Dr. Md. Latiful Bari, Chief Scientist and Head of Food Analysis and Research Laboratory, Center for Advance Research in Sciences, University of Dhaka, Bangladesh for his scientific advice during the study. Views and opinions expressed in this study are those of the authors views; the Asian Journal of Agriculture and Rural Development shall not be responsible or answerable for any loss, damage, or liability, etc. caused in relation to/arising out of the use of the content. 


\section{REFERENCES}

Akhter, S., Naz, S., Sultan, T. M., Mahmood, S., Nasir, M., \& Ahmad, A. (2010). Physicochemical attributes and heavy metal content of mangoes (Mangifera indicaL.) cultivated in different regions of Pakistan. Pakistan Journal of Botany, 42(4), $2691-2702$.

Almeida, M. M. B., de Sousa, P. H. M., Arriaga, Â. M. C., do Prado, G. M., de Carvalho Magalhães, C. E., Maia, G. A., \& de Lemos, T. L. G. (201 1). Bioactive compounds and antioxidant activity of fresh exotic fruits from northeastern Brazil. Food Research International, 44(7), 2155-2 159.Available at: https://doi.org/10.1016/j.foodres.2011.03.051

AOAC. (2005). Official methods of analysis. Arlington, VA, USA: Association of Official Analytical Chemists.

Aoshima, H., Hirata, S., \& Ayabe, S. (2007). Antioxidative and anti-hydrogen peroxide activities of various herbal teas. Food Chemistry, 103(2), 617-622.Available at: https://doi.org/10.1016/j.foodchem.2006.08.032.

Ara, R., Motalab, M., Uddin, M., Fakhruddin, A., \& Saha, B. (2014). Nutritional evaluation of different mango varieties available in Bangladesh. International Food Research Journal, 21(6), 2169.

Bahadori, M. B., Zengin, G., Bahadori, S., Dinparast, L., \& Movahhedin, N. (2018). Phenolic composition and functional properties of wild mint (Mentha longifolia var. calliantha (Stapf) Briq.). International Journal of Food Properties, 21(1), 183193.Available at: https://doi.org/10.1080/10942912.2018.1440238.

Benzie, I. F. F., \& Strain, J. J. (1996). The ferric reducing ability of plasma (FRAP) as a measure of "antioxidant power": The FRAP assay. Analytical Biochemistry, 239(1), 70-76.Available at: https://doi.org/10.1006/abio.1996.0292.

Bogucka-Kocka, A., Zidorn, C., Kasprzycka, M., Szymczak, G., \& Szewczyk, K. (2018). Phenolic acid content, antioxidant and cytotoxic activities of four Kalanchoe species. Saudi Journal of Biological Sciences, 25(4), 622-630.Available at https://doi.org/10.1016/j.sjbs.2016.01.037.

Brand-Williams, W., Cuvelier, M.-E., \& Berset, C. (1995). Use of a free radical method to evaluate antioxidant activity. LWT-Food Science and Technology, 28(1), 25-30.Available at: https://doi.org/10.1016/s0023-6438(95)80008-5.

Burgos, G., Amoros, W., Muñoa, L., Sosa, P., Cayhualla, E., Sanchez, C., \& Bonierbale, M. H. (2013). Total phenolic, total anthocyanin and phenolic acid concentrations and antioxidant activity of purple-fleshed potatoes as affected by boiling Journal of Food Composition and Analysis, 30(1), 6-12.Available at: https://doi.org/10.1016/j.jfca.2012.12.001.

Chang, C. C., Yang, M. H., Wen, H. M., \& Chern, J. C. (2002). Estimation of total flavonoid content in propolis by two complementary colorimetric methods. Journal of Food and Drug Analysis, 10(3), 178-182.Available at: https://doi.org/10.38212/22246614.2748 .

Dutta, S., \& Ray, S. (2020). Comparative assessment of total phenolic content and in vitro antioxidant activities of bark and leaf methanolic extracts of Manilkara hexandra (Roxb.) Dubard. Journal of King Saud University-Science, 32(1), 643-647.

Elfalleh, W., Tlili, N., Nasri, N., Yahia, Y., Hannachi, H., Chaira, N., \& Ferchichi, A. (2011). Antioxidant capacities of phenolic compounds and tocopherols from Tunisian pomegranate (Punica granatum) fruits. Journal of Food Science, 76(5), C707C713.Available at: https://doi.org/10.1111/j.1750-3841.2011.02179.x.

FAOSTAT. (2019). Statistics division, food and agriculture organization of the United Nations. Rome, Italy: Viale delle Terme di Caracalla 00153.

Guo, H., Saravanakumar, K., \& Wang, M.-H. (2018). Total phenolic, flavonoid contents and free radical scavenging capacity of extracts from tubers of Stachys affinis. Biocatalysis and Agricultural Biotechnology, 15, 235-239.Available at: https://doi.org/10.1016/j.bcab.2018.06.009.

Halliwell, B., \& Gutteridge, J. M. C. (2000). Free radicals in biology and medicine (pp. 617-624). Oxford: Oxford Science Publications, Oxford University Press, England.

Holden, J. M., Eldridge, A. L., Beecher, G. R., Buzzard, I. M., Bhagwat, S., Davis, C. S., \& Schakel, S. (1999). Carotenoid content of US foods: An update of the database. Journal of Food Composition and Analysis, 12(3), 169-196.Available at: https://doi.org/10.1006/jfca.1999.0827.

Jukes, T. H. (1974). Are recommended daily allowances for vitamin C adequate? Proceedings of the National Academy of Sciences, U.S.A, 71(5), 1949-1951.Available at: https://doi.org/10.1073/pnas.71.5.1949.

Kuskoski, E. M., García Asuero, A., Morales Millán, M. T., \& Fett, R. (2006). Tropical wild fruits and frozen fruit pulps: Antioxidant activity, polyphenols and anthocyanins. Rural Science, 36(4), 1283-1287.

Leong, S. Y., \& Oey, I. (2012). Effects of processing on anthocyanins, carotenoids and vitamin C in summer fruits and vegetables. Food Chemistry, 133(4), 1577-1587.Available at: https://doi.org/10.1016/j.foodchem.2012.02.052.

Mitra, S. K., Pathak, P. K., \& Chakraborty, I. (2008). Potential use of underutilized crops for nutritional and medicinal properties. In Smith, J. and Haq, N. (Eds.), New Crops and Uses: Their Role in rapidly Changing world (pp. 357). UK: USouthamption University, Center for Underutilised Crops.

Nelson, N. (1944). A photometric adaptation of the Somogyi method for the determination of glucose. Journal of Biological Chemistry, $153(2), 375-380$

Osorio-Esquivel, O., Álvarez, V. B., Dorantes-Álvarez, L., \& Giusti, M. M. (2011). Phenolics, betacyanins and antioxidant activity in Opuntia joconostle fruits. Food Research International, 44(7), 2160-2168.Available at https://doi.org/10.1016/j.foodres.2011.02.011.

Ough, C. S., \& Amerine, M. A. (1988). Phenolic compounds. In: Methods for analysis of musts and wines. New York, USA: J Wiley and Sons, Inc.

Pandey, A., \& Negi, P. S. (2018). Phytochemical composition, in vitro antioxidant activity and antibacterial mechanisms of Neolamarckia cadamba fruits extracts. Natural Product Research, 32(10), 1189-1192.Available at: https://doi.org/10.1080/14786419.2017.1323209.

Paul, S. E., Chakrabarty, S., Jana, S. C., Hasan, M. A., Mandal, K. K., Sarkar, S., \& Mazumdar, D. (2007). A multivariate approach to study the sensory parameters of guava jelly on the basis of the physico-chemical parameters of guava fruit. Acta Horticulturae, 35, 561-568.

Prieto, P., Pineda, M., \& Aguilar, M. (1999). Spectrophotometric quantitation of antioxidant capacity through the formation of a phosphomolybdenum complex: Specific application to the determination of vitamin E. Analytical Biochemistry, 269(2), 337341.Available at: https://doi.org/10.1006/abio.1999.4019.

Ranganna, S. (1995). Handbook of analysis and quality control for fruit and vegetable products (2nd ed.). New Delhi, India: McGraw Hill.

Sathyanarayanan, S., Chandran, R., Thankarajan, S., Abrahamse, H., \& Thangaraj, P. (2018). Phytochemical composition, antioxidant and anti-bacterial activity of Syzygium calophyllifolium Walp. fruit. Journal of Food Science and Technology, 55(1), 341350.Available at: https://doi.org/10.1007/s 13 197-017-2944-6. 
Shajib, M. T. I., Kawser, M., Miah, M. N., Begum, P., Bhattacharjee, L., Hossain, A., \& Islam, S. N. (2013). Nutritional composition of minor indigenous fruits: Cheapest nutritional source for the rural people of Bangladesh. Food Chemistry, 140(3), 466470.Available at: https://doi.org/10.1016/j.foodchem.2012.11.035.

Shukla, Y., Dubey, S., Jain, S., \& Kumar, S. (2001). Chemistry, biology and uses of Adansonia digitata-a review. Journal of Medicinal and Aromatic Plant Sciences, 23(3), 429-434.

Thaipong, K., Boonprakob, U., Crosby, K., Cisneros-Zevallos, L., \& Byrne, D. H. (2006). Comparison of ABTS, DPPH, FRAP, and ORAC assays for estimating antioxidant activity from guava fruit extracts. Journal of Food Composition and Analysis, 19(67), 669-675.Available at: https://doi.org/10.1016/j.jfca.2006.01.003.

Tiburski, J. H., Rosenthal, A., Deliza, R., de Oliveira Godoy, R. L., \& Pacheco, S. (2011). Nutritional properties of yellow mombin (Spondias mombin L.) pulp. Food Research International, 44(7), 2326-2331.Available at https://doi.org/10.1016/j.foodres.2011.03.037.

Wu, X., Beecher, G. R., Holden, J. M., Haytowitz, D. B., Gebhardt, S. E., \& Prior, R. L. (2006). Concentrations of anthocyanins in common foods in the United States and estimation of normal consumption. Journal of Agricultural and Food Chemistry, 54(11), 4069-4075.Available at: https://doi.org/10.1021/jfo6030ol. 